\section{Contribution of Imaging to Organs at Risk Dose during Lung Stereotactic Body Radiation Therapy}

\author{
Milovan Savanović1,2*(e), François Gardavaud³ ${ }^{3}$ Dražan Jaroš4, \\ Bénédicte Lonkuta5 ${ }^{5}$ Matthias Barral ${ }^{6}$, François Henri Cornelis7, \\ Jean-Noël Foulquier ${ }^{8}$
}

\begin{abstract}
Background: The use of imaging is indispensable in modern radiation therapy, both for simulation and treatment delivery. For safe and sure utilization, dose delivery from imaging must be evaluated.

Objective: This study aims to investigate the dose to organ at risk (OAR) delivered by imaging during lung stereotactic body radiation therapy (SBRT) and to evaluate its contribution to the treatment total dose.
\end{abstract}

Material and Methods: In this retrospectively study, imaging total dose to organs at risk (OARs) (spinal cord, esophagus, lungs, and heart) and effective dose were retrospectively evaluated from 100 consecutive patients of a single institution who had lung SBRT. For each patient, dose was estimated using Monte-Carlo convolution for helical computed tomography (helical CT), Four-Dimensional CT (4D$\mathrm{CT})$, and kilovoltage Cone-Beam CT (kV-CBCT). Helical CT and kV-CBCT dose were evaluated for the entire thorax acquisition, while 4D-CT dose was analyzed on upper lobe (UL) or lower lobe (LL) acquisition. Treatment dose was extracted from treatment planning system and compared to imaging total dose.

Results: Imaging total dose maximum values were $117 \mathrm{mGy}$ to the spinal cord, $127 \mathrm{mGy}$ to the esophagus, $176 \mathrm{mGy}$ to the lungs and $193 \mathrm{mGy}$ to the heart. The maximum effective dose was $19.65 \mathrm{mSv}$ for helical CT, $10.62 \mathrm{mSv}$ for $\mathrm{kV}-\mathrm{CBCT}$, $25.95 \mathrm{mSv}$ and $38.45 \mathrm{mSv}$ for 4D-CT in UL and LL regions, respectively. Depending on OAR, treatment total dose was higher from 1.7 to 8.2 times than imaging total dose. Imaging total dose contributed only to $0.3 \%$ of treatment total dose.

Conclusion: Imaging dose delivered with 4D-CT to the OARs is higher than those of others modalities. The heart received the highest imaging dose for both UL and LL. Total imaging dose is negligible since it contributed only to $0.3 \%$ of treatment total dose.

Citation: Savanović M, Gardavaud F, Jaroš D, Lonkuta B, Barral M, Cornelis FH, Foulquier JN. Contribution of Imaging to Organs at Risk Dose during Lung Stereotactic Body Radiation Therapy. J Biomed Phys Eng. 2021;11(2):125-134. doi: 10.31661/jbpe.v0i0.2009-1173.

\section{Keywords}

Lung; Organs at Risk Exposure; Computed Tomography; Four-Dimensional Computed Tomography; Cone-Beam Computed Tomography; Stereotactic Body Radiation Therapy

\section{Introduction}

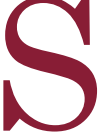
tereotactic Body Radiation Therapy (SBRT) of lung cancer uses high doses delivered into a tumor in few fractions [1]. Since SBRT uses high doses, it is necessary to minimize treatment uncertainties performing conformal targeting, precise tumor and organ delineation, and patient positioning to ensure tumor local control and to limit
${ }^{1}$ PhD Candidate, Department of Radiation Oncol-

ogy, Tenon Hospital,

75020 Paris, France

${ }^{2} \mathrm{PhD}$ Candidate, Faculty

of Medicine, University

of Paris-Saclay, 94276

Le Kremlin-Bicêtre,

France

${ }^{3}$ PhD Candidate, De-

partment of Radiology,

Tenon Hospital, 75020

Paris, France

${ }^{4} \mathrm{PhD}$ Candidate, Affidea,

International Medical

Centers, Center for

Radiotherapy, 78000

Banja Luka, Bosnia and

Herzegovina

${ }^{5} \mathrm{MSc}$, Department of Ra-

diology, Tenon Hospital,

75020 Paris, France

${ }^{6} \mathrm{MD}$, Department of Ra-

diology, Tenon Hospital,

75020 Paris, France

${ }^{7} \mathrm{PhD}$, Department of Ra-

diology, Tenon Hospital,

75020 Paris, France

8PhD, Department of

Radiation Oncology,

Tenon Hospital, 75020

Paris, France

*Corresponding author:

Milovan Savanovic

Department of Radiation

Oncology, Tenon Hospi-

tal, 75020 Paris, France

E-mail: milovan_sa-

vanovic@yahoo.com

Received: 1 September 2020 Accepted: 7 November 2020 
organs at risk (OAR) radiation exposure. In lung SBRT, the computed tomography (CT) in helical mode (helical CT) and Four-Dimensional CT (4D-CT) are used during simulation [2]. To ensure patient positioning during treatment delivery, kilovoltage Cone-Beam CT $(\mathrm{kV}-\mathrm{CBCT})$ is recommended to reduce uncertainties due to patient positioning, inter- and intra-fraction motion [3-5].

However, image guidance has an important role in improving SBRT safety and efficacy, it could yield to expose OARs outside the therapeutic beams $[6,7]$. If significant image guidance dose (generally using MV imaging) can theoretically have an impact on tumor and OARs biological effects such as local control, necrosis or tissue damage [8-11]. $\mathrm{kV}$ imaging may increase the secondary cancer risk, particularly for organs outside the treatment field [12-14]. According to the American Association of Physics in Medicine (AAPM), imaging dose should be included in the treatment planning when superior to $5 \%$ of planned dose [6]. It requires to consider dose calculation and delivery accuracy, tumor-to-organ proximity, dose tolerances for critical organs, treatment technique and feasibility in clinical practice to ensure tumor local control and better sparing of OARs [6].

Only three studies, two with $\mathrm{kV}-\mathrm{CBCT}$ and one with 4D-CT, have evaluated imaging dose with adult patients with lung cancer [15-17]. In the present study, patient-specific imaging dose was evaluated for lung SBRT, including dose delivered during simulation using helical CT and 4D-CT, and before each fraction of treatment delivery using $\mathrm{kV}-\mathrm{CBCT}$ [18]. The purpose of this study was to investigate the dose to OAR delivered by imaging during lung SBRT and to evaluate its contribution to the treatment total dose.

\section{Material and Methods}

\section{Patient selection}

In this retrospectively study, a total of 100 patients, with small tumor size $(\leq 5 \mathrm{~cm})$ who had lung SBRT, were included. The patients' characteristics are presented in Table 1.

All patients were enrolled in an Institutional Review Board approved protocol. This protocol was Health Insurance Portability and Accountability Act compliant.

CT acquisitions and dose calculation

During simulation for lung SBRT, patients were positioned in the supine position with arms above the head using the BlueBAG BodyFIX system immobilization (Medical Intelligence, Schwabmünchen, Germany). All patients were scanned on 16 slices CT scan (GE Lightspeed, General Electric Medical Systems, Waukesha, WI, USA), equipped with the Real-time Positioning Management system (RPM, Varian Medical Systems, Palo Alto, CA, USA). From Institutional protocol,

Table 1: Patient characteristics

\begin{tabular}{cc} 
Characteristics & Raw data \\
\hline Number of patients & 100 \\
\hline Age & $70(38-90)$ \\
\hline Gender & 58 \\
\hline Male & 42 \\
\hline Female & \\
\hline Tumor location & 68 \\
\hline Upper lobe & 32 \\
\hline Lower lobe & \\
\hline Tumor size & $32(17-50)$ \\
\hline Upper lobe (mm) & $37(21-49)$ \\
\hline Lower lobe (mm) & $63.5(39.0-106.0)$ \\
\hline Weight (kg) & $1.68(1.48-1.86)$ \\
\hline Height (m) & $23.0(15.2-35.9)$ \\
\hline BMI (kg/m $\left.{ }^{2}\right)$ & $4.3(1.7-9.6)$ \\
\hline Breathing cycle (s) & $54(29-94)$ \\
\hline 4D-CT scan time (s) & $5(4-8)$
\end{tabular}

BMI: Body mass index, 4D-CT: Four-Dimensional Computed Tomography 
two helical scans were performed, followed by one 4D-CT scan. The CT parameters used during clinical acquisitions are presented in (Table 2).

For the helical scans, acquisition was performed from the cricoid cartilage to the second lumbar vertebra (Figure 1(a)), while 4D$\mathrm{CT}$ acquisition was performed with fifteen slices below and above tumor localization (in the upper lobe (UL) Figure 1 (b) and the lower lobe (LL) region Figure 1 (c)).
For all CT acquisitions, volume computed tomography dose index $\left(\mathrm{CTDI}_{\mathrm{vol}}\right)$ has been extracted automatically from DICOM images using dedicated home-made scripts in MATLAB R2020a (MathWorks, Natick, Massachusetts).

Dose to OARs (spinal cord, esophagus, lungs, heart) was calculated for each patient (see patient anatomical attributes in Table 1) with CT scans parameters (Table 2), in the UL and LL (Figure 2) using the National Cancer Institute dosimetry system for CT (NCICT

Table 2: Parameters of helical computed tomography (CT) and Four-Dimensional Computed Tomography (4D-CT) acquisitions

\begin{tabular}{ccc} 
Parameters & Helical CT & 4D-CT \\
\hline Rotation time (s) & 0.7 & 0.7 \\
\hline Pitch & 0.938 & $/$ \\
\hline Images per rotation & $/$ & 16 \\
\hline Collimation $(\mathbf{m m})$ & 20 & 20 \\
\hline Slice thickness $(\mathbf{m m})$ & 1.25 & 1.25 \\
\hline SFOV & Large Body & Large Body \\
\hline kV & 120 & 120 \\
\hline mA min & 10 & 10 \\
\hline mA max & 440 & 440 \\
\hline Noise index & 9.83 & 9.83 \\
\hline AutomA/smartmA & Yes / Yes & Yes / Yes
\end{tabular}

CT: Computed tomography, 4D-CT: Four-Dimensional Computed Tomography, SFOV: Scan field of view, $\mathrm{kV}$ : kilo voltage, min $\mathrm{mA}$ : minimum value of milliampere modulation, max $\mathrm{mA}$ : maximum value of milliampere modulation
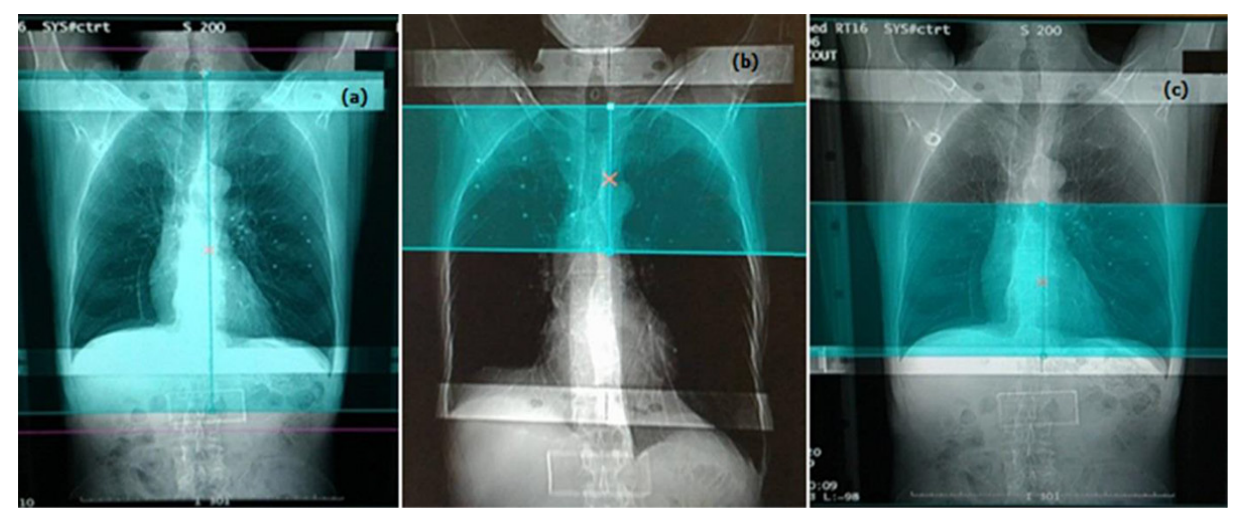

Figure 1: Scan area (in blue box) for helical computed tomography (CT) (a), Four-Dimensional Computed Tomography (4D-CT) acquisition for tumor located in the upper lobe (UL) (b), and 4D-CT acquisition for tumor located in the lower lobe (LL) (c) 


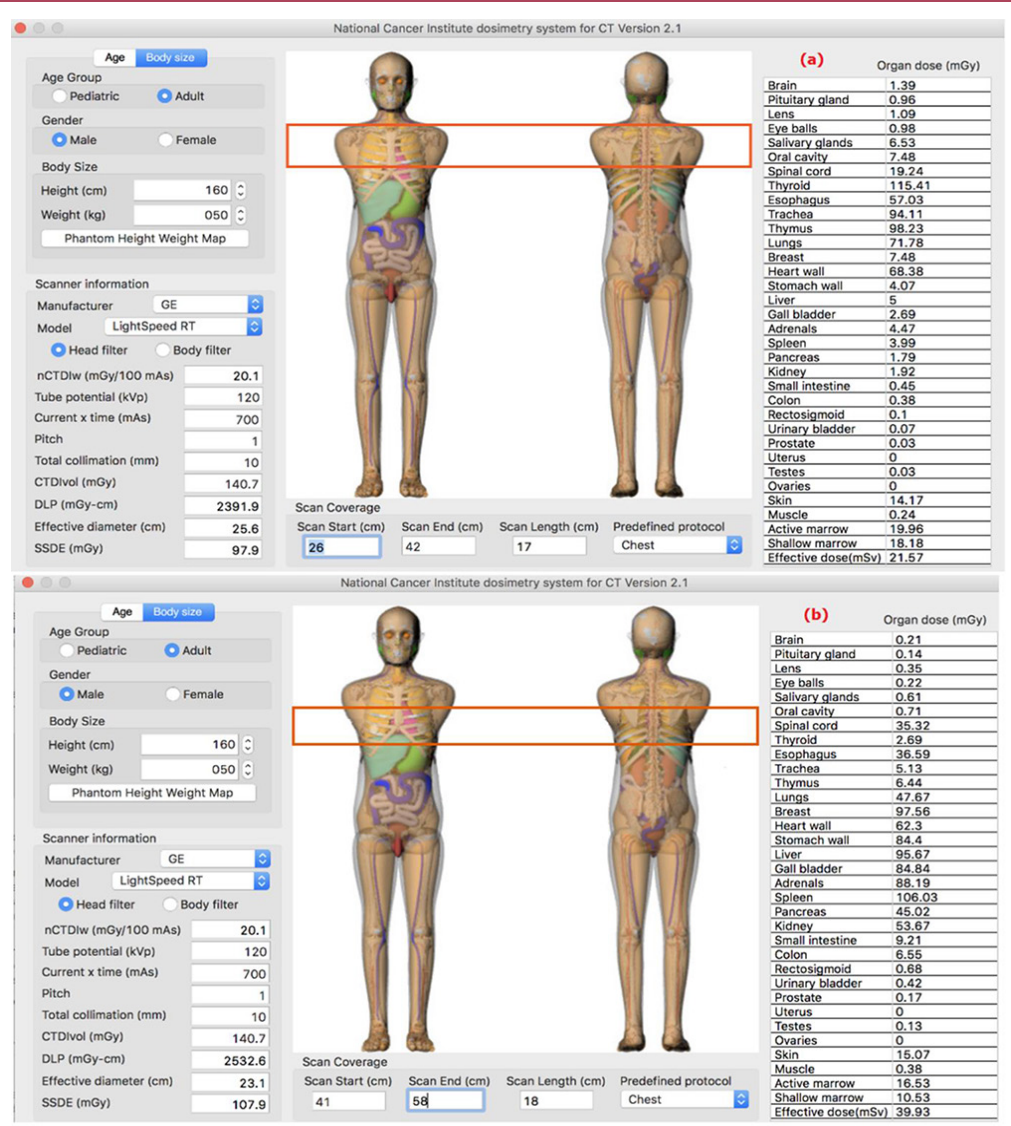

Figure 2: National Cancer Institute dosimetry system for CT (NCICT) software Graphical User Interface (GUI) calculating dose delivered by Four-Dimensional Computed Tomography (4D-CT) to organs at risk (OARs) in the scan area illustrated with the red rectangle for upper lobe (UL) (a) and lower lobe (LL) regions (b)

v2.1, NIH, Bethesda, Maryland, USA) based on Monte-Carlo convolution [19]. Helical CT dose simulated in NCICT included the complete thorax (Figure 1 (a)), whereas 4DCT examination scan area depends on tumor location: UL or LL (Figure 2). The scanned region size was different for each patient (in regard of the tumor size) and the patient body habitus was tailored for each patient in NCICT thanks to the International Commission on Radiological Protection (ICRP) reference adult phantoms [20, 21]. All the scan parameters displayed in Table 1 were implemented in NCICT software to determine organ dose exposure, except for tube current modulation (TCM) where the miliAmpere mean value, specific for each exam. This was retained since NCICT v2.1 does not offer an accessible option in the Graphical User Interface (GUI) to take into account TCM mode.

kV-CBCT acquisitions and dose calculation

During treatment delivery with a Novalis TrueBeam STx (Varian Medical System, Palo Alto, CA, USA), the patient positioning was verified using $\mathrm{kV}$-CBCT scan. The $\mathrm{kV}$-CBCT images were acquired with half-fan full scan mode $\left(360^{\circ}\right), 125 \mathrm{kV}, 270 \mathrm{mAs}, 14^{\circ}$ anode angle, field size $20 \mathrm{~cm} \times 26.5 \mathrm{~cm}, 900$ projections and $60 \mathrm{~s}$ for the exposure time. For all $\mathrm{kV}-\mathrm{CBCT}$ acquisitions, volume computed tomography dose index $\left(\mathrm{CTDI}_{\mathrm{vol}}\right)$ has been extracted automatically from DICOM images using dedicated home-made scripts in MATLAB R2020a (MathWorks, Natick, Massachusetts).

To measured air kerma, the half-value layer 
(HVL) and the beam filtration, Black Piranha detector (RTI Electronics Inc, NJ, USA) was used. Then a Monte Carlo program for calculating patient organ doses in medical x-ray examinations (PCXMC v2.0, Stuk, Helsinki, Finland) was used in batch mode to compute dose delivered to the OARs from $\mathrm{kV}$-CBCT acquisitions [22]. Each patient body habitus has been tailored in PCXMC software.

Calculation of total exposure from imaging acquisitions

Imaging total dose delivered to the OARs is calculated as a total sum of doses delivered to the OARs from all modalities: helical CT scan, 4D-CT scan and $\mathrm{kV}$-CBCT during lung SBRT treatment. For the helical CT and kVCBCT scans, imaging dose evaluation was performed for the entire thorax, while imaging dose from 4D-CT was evaluated for specific scanned regions such as UL or LL (red rectangles in Figure 2).

Imaging total dose was calculated based on institutional protocol, by summing doses from two helical CT scans, one cine 4D-CT and five $\mathrm{kV}-\mathrm{CBCT}$ (five being the mean value of all fractions).

Imaging total dose delivered to one organ was presented by equation (1):

$D_{t}\left(\operatorname{organ}_{i}\right)=\left(a \times D_{h C T}\left(\operatorname{organ}_{i}\right)\right)+D_{4 D-C T}\left(\operatorname{organ}_{i}\right)+\left(b \times D_{C B C T}\left(\operatorname{organ}_{i}\right)(1)\right.$

Where $D_{t}\left(\right.$ organ $\left._{i}\right)$-imaging total dose delivered to the organ of interest, $a$ - number of helical CT sequences, $D_{h C T}\left(\right.$ organ $\left._{i}\right)-$ dose delivered by helical $\mathrm{CT}$ to the organ of interest, $D_{4 D-C T}\left(\right.$ organ $\left._{i}\right)$ - dose delivered by $4 \mathrm{D}-\mathrm{CT}$ to the organ of interest, $b$ - number of CBCT sequences, $D_{C B C T}\left(\right.$ organ $\left._{i}\right)$ - dose delivered by CBCT to the organ of interest.

For each OAR, the maximum value of imaging total dose has been computed.

Treatment planning and dose constraints

Lung SBRT plans were performed on the helical CT scan without stereotactic body frame with two to four partial dynamic conformal arcs (DCAs) using $6 \mathrm{MV}$ beams. The treat- ment dose was 60 Gy in 4 to 8 fractions prescribed to isodose line $80 \%$. Treatment planning was performed with the Pinnacle Version 9.10 (Koninklijke Philips N.V., Amsterdam, Netherlands) treatment planning system (TPS) and calculated using the Collapsed Cone Convolution (CCC) algorithm [23, 24]. The constraints for the planning target volume (PTV) coverage were D98\%>95\% and D95\% $\geq 100 \%$. The OARs constraints were based on ICRU 91 report recommendations using maximum dose $\left(\mathrm{D}_{\max }\right)$ which should be less than $15 \mathrm{~Gy}(0.1 \mathrm{cc})$ for the spinal cord, $30 \mathrm{~Gy}(0.5 \mathrm{cc})$ for the heart and $27 \mathrm{~Gy}(0.5 \mathrm{cc})$ for the esophagus [25]. For the lungs volume, where the PTV had been subtracted (Lungs-PTV), two constraints were established. The percentage volume receiving 20 Gy or more $\left(\mathrm{V}_{20}\right)$ and 16 Gy or more $\left(\mathrm{V}_{16}\right)$ was restricted to $10 \%$ and $20 \%$ of the prescribed dose respectively.

Dose-volume histograms (DVH) were generated for the PTV and all OARs. OARs doses were evaluated using mean dose $\left(\mathrm{D}_{\text {mean }}\right)$ and $\mathrm{D}_{\max }$, respectively. Doses delivered to the OARs were manually extracted from DVHs for all 100 patients.

\section{Data analysis}

Statistical analysis and violin plots computation of doses delivered to the OARs (spinal cord, esophagus, lungs and heart) from imaging chain were evaluated using GraphPad Prism 8.1.2 version (SD, California, USA). To compare results between all modalities, Kruskal Wallis test was used. The imaging maximum total dose has been compared to the treatment total dose using paired t-test. Data was considered statistically significant at $p<$ 0.05 .

\section{Results}

The mean values of the CTDI ${ }_{\text {vol }}$ obtained were $23.1 \pm 3.8 \mathrm{mGy}$ and $55.6 \pm 13.3 \mathrm{mGy}$ $(\mathrm{p}<0.0001)$ during helical CT and cine 4D-CT scans, respectively, while $\mathrm{CTDI}_{\mathrm{vol}}$ from $\mathrm{kV}$ $\mathrm{CBCT}$ remained constant at $4 \mathrm{mGy}$. Air kerma 
mean value was $13.8 \pm 0.3 \mathrm{mGy}$. Mean halfvalue layer was $4.88 \pm 0.01 \mathrm{mmAl}$ and mean beam filtration was $10.12 \pm 0.01 \mathrm{mmAl}$. Imaging mean dose delivered to the OARs and associated effective dose are presented in Table 3 . Results presented in Table 3 were all statistically significant $(\mathrm{p}<0.01)$.

Imaging doses maximum values delivered to the OARs from helical CT, $\mathrm{kV}-\mathrm{CBCT}$ and 4D$\mathrm{CT}$ in the UL and LL area were, respectively: $19.52 \mathrm{mGy}, 9.74 \mathrm{mGy}, 37.76 \mathrm{mGy}$ and 36.89 mGy to the spinal cord; $29.74 \mathrm{mGy}, 5.03 \mathrm{mGy}$, $56.69 \mathrm{mGy}$ and $36.95 \mathrm{mGy}$ to the esophagus; $40.01 \mathrm{mGy}, 6.32 \mathrm{mGy}, 83.48 \mathrm{mGy}$ and 50.58 mGy to the lung; $41.05 \mathrm{mGy}, 9.13 \mathrm{mGy}, 68.60$ $\mathrm{mGy}$ and $66.66 \mathrm{mGy}$ to the heart.

Imaging total dose maximum values were $117 \mathrm{mGy}$ to the spinal cord, $127 \mathrm{mGy}$ to the esophagus, $176 \mathrm{mGy}$ to the lungs and 193 mGy to the heart.

The maximum effective dose was 19.65 $\mathrm{mSv}$ for helical CT, $10.62 \mathrm{mSv}$ for $\mathrm{kV}$-CBCT, $25.95 \mathrm{mSv}$ and $38.45 \mathrm{mSv}$ for 4D-CT in UL and LL regions, respectively.

The highest imaging doses were delivered to the heart (41.05 mGy) for helical CT $(p<0.0001)$, to the spinal cord $(9.74 \mathrm{mGy})$ for $\mathrm{kV}$-CBCT $(\mathrm{p}<0.0001)$, to the lung $(83.48$ $\mathrm{mGy}$ ) for 4D-CT in UL region ( $<<0.0001)$ and to the heart (68.60 mGy) for 4D-CT in LL re- gion $(\mathrm{p}<0.0001)$.

Imaging doses delivered to the OARs (spinal cord, esophagus, lungs and heart) were presented in Figure 3.

The PTV coverage (D98\% $>95 \%$ was $98.5 \pm 0.6 \%$ and $\mathrm{D} 95 \% \geq 100 \% 100.06 \pm 1.1 \%)$ yields to OARs doses. Mean doses $\left(\mathrm{D}_{\text {mean }}\right)$ and maximum doses $\left(\mathrm{D}_{\max }\right)$ in volume $(0.1 \mathrm{cc}$ for the spinal cord and $0.5 \mathrm{cc}$ for the heart and esophagus) delivered to the OARs were presented depending on the lobe (UL and LL regions) in Table 4.

Comparison of treatment beam mean doses with imaging total mean dose, depending on UL and LL regions, was presented in Table 5 with associated relative gap rations (RGR) and p-values.

\section{Discussion}

Imaging total dose was evaluated as the sum of the doses delivered to the OARs from two helical CT scans, one 4D-CT scan and five $\mathrm{kV}$ CBCT acquisitions. Imaging maximum total dose was delivered to the heart (193 mGy), then to the lungs (176 mGy), the esophagus (127 mGy) and the spinal cord (117 mGy). These doses are negligible comparing to the total dose delivered during SBRT treatment for lung cancer. Indeed, imaging total dose contributed up to only $0.3 \%$ of 60 Gy of the treat-

Table 3: Mean values and standard deviation of the dose delivered to the organs at risk (OARs), effective dose and imaging total dose were evaluated from different modalities depending on scanned regions

\begin{tabular}{ccccccc} 
Technique & Zone & $\begin{array}{c}\text { Spinal cord } \\
(\mathbf{m G y})\end{array}$ & $\begin{array}{c}\text { Esophagus } \\
(\mathbf{m G y})\end{array}$ & Lungs $(\mathbf{m G y})$ & Heart $(\mathbf{m G y})$ & $\mathbf{D}_{\text {eff }}(\mathbf{m S v})$ \\
\hline Helical CT & WT & $11.54 \pm 3.05$ & $21.08 \pm 4.30$ & $29.02 \pm 5.40$ & $29.40 \pm 5.76$ & $14.29 \pm 2.43$ \\
\hline kV-CBCT & WT & $7.14 \pm 1.46$ & $3.52 \pm 1.66$ & $4.50 \pm 2.11$ & $6.86 \pm 2.41$ & $1.33 \pm 0.63$ \\
\hline \multirow{2}{*}{ 4D-CT } & UL & $11.80 \pm 5.40$ & $32.07 \pm 8.19$ & $46.45 \pm 12.67$ & $38.44 \pm 12.07$ & $13.93 \pm 4.11$ \\
\cline { 2 - 7 } & LL & $17.77 \pm 6.64$ & $21.67 \pm 6.77$ & $30.20 \pm 9.42$ & $39.69 \pm 12.46$ & $22.43 \pm 6.04$ \\
\hline \multirow{2}{*}{ Total } & UL & $74.40 \pm 15.72$ & $94.47 \pm 13.87$ & $130.14 \pm 19.19$ & $135.97 \pm 20.89$ & $50.28 \pm 6.62$ \\
\cline { 2 - 6 } & LL & $77.26 \pm 16.59$ & $80.29 \pm 14.77$ & $109.57 \pm 18.67$ & $131.91 \pm 23.59$ & $56.95 \pm 8.66$
\end{tabular}

CT: Computed tomography, kV-CBCT: kilovoltage Cone-Beam computed tomography, 4D-CT: Four-Dimensional Computed Tomography, WT: Whole thorax, UL: Upper lobe, LL: Lower lobe, mGy: milli-gray, $\mathrm{D}_{\text {eff }}$ : Effective dose 

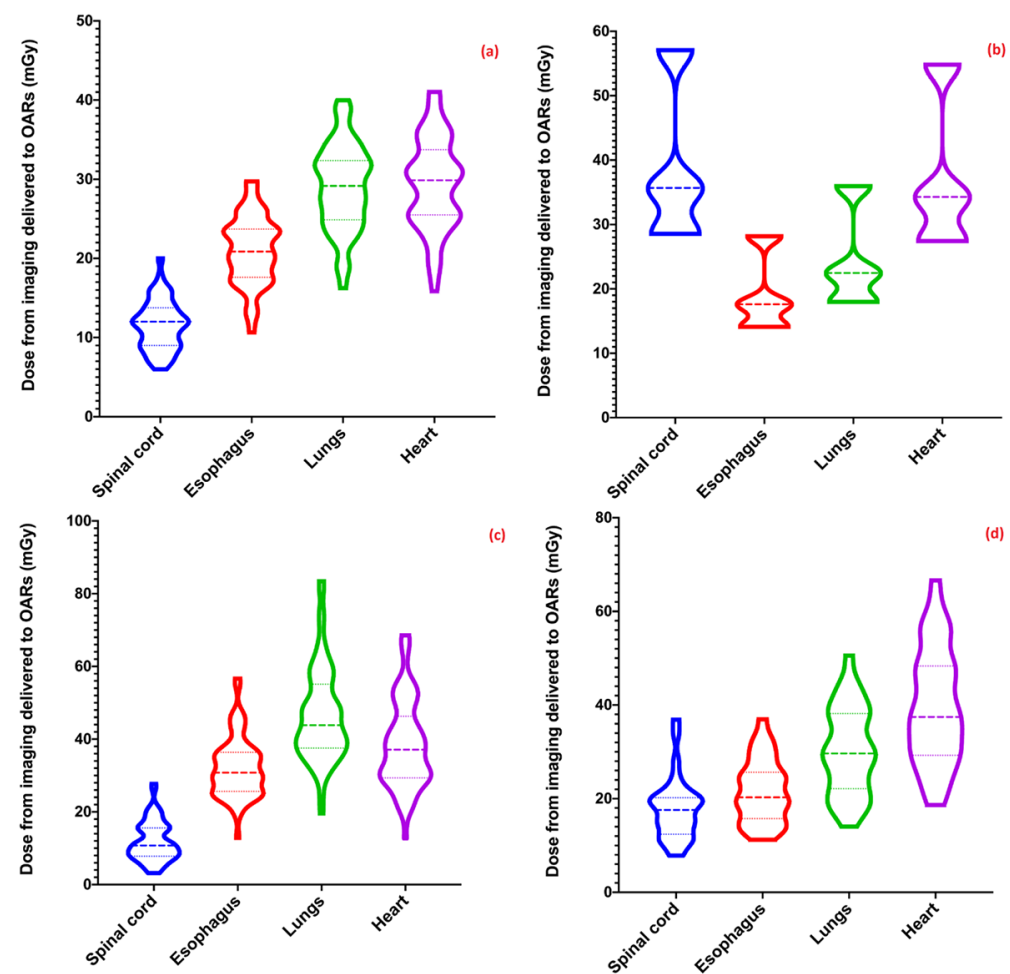

Figure 3: Violin plots of dose delivered to the organs at risk (OARs) evaluated from Helical computed tomography (CT) from whole thorax (a); kilovoltage Cone-Beam CT (kV-CBCT) from whole thorax (b); cine Four-Dimensional Computed Tomography (4D-CT) in upper lobe (UL) region (c); and cine 4D-CT in lower lobe (LL) area (d). The dashed lines near the middle of the plots indicate the medians and the dot lines indicate the quartiles

Table 4: The results of mean doses and maximum doses in volume $(0.1 \mathrm{cc}$ for the spinal cord and $0.5 \mathrm{cc}$ for the heart and esophagus) evaluated from treatment planning system (TPS) for the spinal cord, esophagus, lungs and heart, depending on the lobe (upper lobe (UL) and lower lobe (LL) regions)

\begin{tabular}{|c|c|c|c|c|c|c|}
\hline \multirow{2}{*}{ Lobe } & \multirow{2}{*}{ Dose } & \multirow{2}{*}{ Spinal cord } & \multirow{2}{*}{ Esophagus } & \multicolumn{2}{|c|}{ Lungs } & \multirow{2}{*}{ Heart } \\
\hline & & & & $V_{20}<10 \%$ & $V_{16}<20 \%$ & \\
\hline \multirow{2}{*}{ UL } & $\mathrm{D}_{\text {mean }}(\mathrm{Gy})$ & $1.24 \pm 0.93$ & $2.84 \pm 2.42$ & $3.49 \pm 1.84$ & \multirow{2}{*}{$6.05 \pm 3.96$} & $0.93 \pm 1.86$ \\
\hline & $\mathrm{D}_{\text {max }}(\mathrm{Gy})$ & $10.19 \pm 6.31$ & $14.93 \pm 11.97$ & $4.62 \pm 3.13$ & & $8.74 \pm 16.18$ \\
\hline \multirow{2}{*}{ LL } & $\mathrm{D}_{\text {mean }}(\mathrm{Gy})$ & $1.54 \pm 1.27$ & $2.52 \pm 1.33$ & $3.88 \pm 2.37$ & \multirow{2}{*}{$6.84 \pm 4.42$} & $3.27 \pm 3.35$ \\
\hline & $\mathrm{D}_{\max }(\mathrm{Gy})$ & $11.02 \pm 6.20$ & $10.94 \pm 3.64$ & $5.11 \pm 3.46$ & & $16.54 \pm 14.51$ \\
\hline
\end{tabular}

$\mathrm{UL}$ : Upper lobe, LL: Lower lobe, $\mathrm{D}_{\text {mean }}$ : mean dose, $\mathrm{D}_{\max }$ : maximum dose

ment total dose delivered. It is worth to consider that imaging total dose delivered to the OARs is insufficient to impact on tumor local control (necrosis) and/or OARs damage, because it represents less than $5 \%$ of prescribed treatment dose according to AAPM [6]. This result is comparable with data currently available in the literature which indicates that imaging dose during image guidance is generally less than $5 \%$ of the therapeutic target dose 
Table 5: Comparison between treatment beam mean doses and imaging total mean dose delivered to the organs at risk (OARs) depending on upper lobe (UL) and lower lobe (LL) areas with associated relative gap rations and $p$-values were presented

\begin{tabular}{ccccccccc} 
& \multicolumn{4}{c}{ Upper lobe } & \multicolumn{5}{c}{ Lower lobe } \\
\cline { 2 - 9 } OARs & TPS & Imaging & RGR & $\mathbf{p}$ & TPS & Imaging & RGR & $\mathbf{p}$ \\
\cline { 2 - 9 } & $(\mathrm{mGy})$ & $(\mathrm{mGy})$ & & & $(\mathrm{mGy})$ & $(\mathrm{mGy})$ & \\
\hline Sc & $1240 \pm 930$ & $74.4 \pm 15.7$ & 16.7 & $<0.0001$ & $11020 \pm 6200$ & $77.3 \pm 16.6$ & 121 & $<0.0001$ \\
\hline Esophagus & $2840 \pm 2420$ & $94.5 \pm 13.9$ & 30.1 & $<0.0001$ & $10940 \pm 3640$ & $80.3 \pm 14.8$ & 137.6 & $<0.0001$ \\
\hline Lung & $3490 \pm 760$ & $130.1 \pm 19.2$ & 26.8 & $<0.0001$ & $6840 \pm 4420$ & $109.6 \pm 18.7$ & 52.9 & $<0.0001$ \\
\hline Heart & $930 \pm 1840$ & $136.0 \pm 20.9$ & 6.8 & $<0.0001$ & $16540 \pm 14510$ & $131.9 \pm 23.6$ & 106.9 & $<0.0001$
\end{tabular}

OARs: Organs at risk, Sc: Spinal cord, TPS: Treatment planning system, RGR: Relative GAP

\section{[26-28].}

However, as demonstrated in this study, dose delivered to the OARs depends on imaging modality. Highest doses were delivered to the heart from the helical CT $(p<0.0001)$, to the spinal cord from the $\mathrm{kV}-\mathrm{CBCT}(\mathrm{p}<0.0001)$, to the lungs in UL region from $4 \mathrm{D}-\mathrm{CT}$ $(p<0.0001)$, and to the heart in LL region from 4D-CT $(p<0.0001)$. As the heart is located in chest middle, it was the most exposed organ for helical CT, 4D-CT in LL area and for the imaging total dose. For the same reason, it was also the second most exposed for $\mathrm{kV}-\mathrm{CBCT}$ (after spinal cord) and for 4D-CT in the UL (after lungs). The highest dose delivered to the spinal cord from $\mathrm{kV}-\mathrm{CBCT}$ was due to sequential acquisition technique without cradle displacement contrary to helical $\mathrm{CT}$ acquisition which involves cradle displacement. Lungs were the most exposed organ in the UL from 4D-CT due to larger exposed volume than the heart (Figure 1).

In the meantime, higher dose was delivered to the OARs with 4D-CT than helical CT. Comparing helical CT vs 4D-CT acquisitions, higher dose in UL region was delivered with 4D-CT to the esophagus (32\%), to the lung $(33 \%)$ and to the heart $(20 \%)$, while helical CT was delivered higher dose to the spinal cord (9\%) than 4D-CT. In LL region, 4D-CT was delivered higher dose to the spinal cord (33\%) than helical CT, while helical CT was delivered higher dose to the heart $(20 \%)$. A smaller difference between dose delivered to the esophagus (3\%) and lung (2\%) was found comparing helical CT vs 4D-CT in LL area. These doses differences from several modalities could be explained by the fact that the helical CT was performed for the whole thorax region, while 4D-CT was performed on a tailored area depending on tumor localization (UL and LL) (Figure 1). In Yang et al. study, doses to OARs were compared between helical CT scan versus 4D-CT scan [17]. They reported that higher doses were delivered to OARs using 4D-CT compared to helical CT: heart 8.0 vs $103.0 \mathrm{mGy}$; bilateral lungs 7.1 vs $94.6 \mathrm{mGy}$; spinal cord 7.4 vs $94.2 \mathrm{mGy}$; esophagus 7.9 vs 103.7 mGy [17]. Compared to the results from the Yang et al. study, higher doses were delivered to the OARs (from 1.5 to 3.5 times) for the helical CT, while lower doses were delivered to the OARs (from 2 to 5 times) for 4D-CT in this study [17]. These differences were provided from different manufactured CT scans, acquisition parameters (helical CT and 4D-CT) and scanned regions (4D-CT) in this study, compared to the previously cited study [17].

Comparing helical CT versus $\mathrm{kV}-\mathrm{CBCT}$, dose delivered to the OARs was higher using helical CT: 1.7 times to the spinal cord, 5.9 times to esophagus, 6.5 times to the lungs, 4.4 times to the heart. Helical CT delivered 
higher effective dose (10.7 times higher) than $\mathrm{kV}-\mathrm{CBCT}$. Spezi et al. evaluated dose to the lung and spinal cord from six chest cases using the X-ray volume imaging unit mounted on an Elekta Synergy linear accelerator (Elekta, Crawley, UK) [15]. They reported that dose delivered to the lung and spinal cord was less than 40 and $50 \mathrm{mGy}$, respectively [15]. In Nakamura et al. study, imaging mean doses were less than $26 \mathrm{mGy}$ for soft tissues (such as lung, spinal cord and heart) from 3D-CBCT scans for nine lung cancer patients [16]. These OARs received slightly higher mean doses in these previous studies due to differences between their imaging acquisition parameters and those of this study [16].

This study has several limitations. Thus, therapeutic doses were compared to imaging dose by mean values instead of maximum values because commercial imaging dosimetric simulation software did not allow users to perform dose computation on each phantom voxels. In these imaging dosimetric software, only the mean dose was available. Finally, biological effects on exposed organs between low energy $(\mathrm{kV})$ and high energy (MV) could be different as this depends on the secondary electrons range. In the diagnostic imaging field, the secondary electrons range is short due to low energies. At a megavoltage photon beam, the secondary electrons range increases leading to regions where dose buildup is not in electronic equilibrium and thus raises biological risk [29].

\section{Conclusion}

In conclusion, imaging exposure depends on acquisition parameters specific to each imaging modality. OARs exposure was higher using 4D-CT than other imaging modalities and depends on tumor size and location. The imaging maximum total dose compared to total dose delivered from lung SBRT treatment $(0.3 \%)$ was below the AAPM recommended threshold (5\%) and could not be included in treatment planning.

\section{Conflict of Interest}

None

\section{References}

1. Abreu CE, Ferreira PP, De Moraes FY, et al. Stereotactic body radiotherapy in lung cancer: an update. J Bras Pneumol. 2015;41(4):376-87. doi: 10.1590/ S1806-37132015000000034. PubMed PMID: 26398758. PubMed PMCID: PMC4635958.

2. Lambrecht M, Sonke JJ, Nestle U, et al. Quality assurance of four-dimensional computed tomography in a multicentre trial of stereotactic body radiotherapy of centrally located lung tumours. Phys Imag Radiat Oncol. 2018;8:57-62. doi: 10.1016/j. phro.2018.10.003.

3. Molitoris JK, Diwanji T, Snider III JW, et al. Optimizing immobilization, margins, and imaging for lung stereotactic body radiation therapy. Trans/ Lung Cancer Res. 2019;8(1):24-31. doi: 10.21037/ tlcr.2018.09.25. PubMed PMID: 30788232. PubMed PMCID: PMC6351403.

4. Srinivasan K. Mohammadi M. Shepherd J. Applications of linac-mounted kilovoltage Cone-beam Computed Tomography in modern radiation therapy: A review. Pol J Radiol. 2014;79:181-93. doi: 10.12659/PJR.890745. PubMed PMID: 25006356. PubMed PMCID: PMC4085117.

5. Goyal S. Kataria T. Image guidance in radiation therapy: techniques and applications. Radiol Res Pract. 2014;2014:1-10. doi: 10.1155/2014/705604. PubMed PMID: 25587445. PubMed PMCID: PMC4281403.

6. Ding GX, Alaei P, Curran B, et al. Image guidance doses delivered during radiotherapy: Quantification, management, and reduction: Report of the AAPM Therapy Physics Committee Task Group 180. Med Phys. 2018;45(5):e84-99. doi: 10.1002/mp.12824. PubMed PMID: 29468678.

7. Guckenberger M, Meyer J, Wilbert J, et al. Conebeam CT based image-guidance for extracranial stereotactic radiotherapy of intrapulmonary tumors. Acta Oncol. 2006;45(7):897-906. doi: 10.1080/02841860600904839. PubMed PMID: 16982556.

8. Brahme A. Dosimetric precision requirements in radiation therapy. Acta Radiol Oncol. 1984;23:37991. doi: 10.3109/02841868409136037. PubMed PMID: 6095609.

9. Dutreix A. When and how can we improve precision in radiotherapy? Radiother Oncol. 1984;2:27592. doi: 10.1016/S0167-8140(84)80070-5.

10. Wambersie A. What accuracy is required and can be achieved in radiation therapy (review of radiobiological and clinical data). Radiochim Acta. 
2001;89:255-64. doi: 10.1524/ract.2001.89.45.255 .

11. Herring DF. The degree of precision required in the radiation dose delivered in cancer radiotherapy. Proc. 3rd ICCR, 1970 British institute of radiology special report; Glasgow, Scotland: British Institute of Radiology; 1971. p. 51-8.

12. Dzierma $Y$, Mikulla $K$, Richter $P$, et al. Imaging dose and secondary cancer risk in image-guided radiotherapy of pediatric patients. Radiat Oncol. 2018;13(1):168. doi: 10.1186/s13014-018-1109-8.

13. Zhou L, Bai S, Zhang YB, et al. Imaging Dose, Cancer Risk and Cost Analysis in Image-guided Radiotherapy of Cancers. Scientific Reports. 2018;8(1):10076. doi: 10.1038/s41598-018-28431-9.

14. Dong Wook K, Weon C, Myonggeun Y. Imaging Doses and Secondary Cancer Risk From Kilovoltage Cone-beam CT in Radiation Therapy. Health Physics. 2013;104:499-503. doi: 10.1097/ HP.0b013e318285c685. PubMed PMID: 23532078.

15. Spezi $E$, Downes $P$, Jarvis $R$, et al. Patient-specific three-dimensional concomitant dose from cone beam computed tomography exposure in image-guided radiotherapy. Int $J$ Radiat Oncol Biol Phys. 2012;83(1):419-26. doi: 10.1016/j. ijrobp.2011.06.1972. PubMed PMID: 22027261.

16. Nakamura $M$, Ishihara $Y$, Matsuo $Y$, et al. Quantification of the $\mathrm{kV} X$-ray imaging dose during real-time tumor tracking and from three- and four-dimensional cone-beam computed tomography in lung cancer patients using a Monte Carlo simulation. J Radiat Res. 2018;59(2):173-81. doi: 10.1093/jrr/rrx098. PubMed PMID: 29385514. PubMed PMCID: PMC5950977.

17. Yang C, Liu R, Ming X, Liu N, Guan Y, Feng Y. Thoracic Organ Doses and Cancer Risk from Low Pitch Helical 4-Dimensional Computed Tomography Scans. Biomed Res Int. 2018;2018:8927290. doi: $10.1155 / 2018 / 8927290$. PubMed PMID: 30345309. PubMed PMCID: PMC6174794.

18. Li R, Han B, Meng B, et al. Clinical implementation of intrafraction cone beam computed tomography imaging during lung tumor stereotactic ablative radiation therapy. Int $J$ Radiat Oncol Biol Phys. 2013;87(5):917-23. doi: 10.1016/j. ijrobp.2013.08.015. PubMed PMID: 24113060. PubMed PMCID: PMC3888501.

19. Lee C, Kim KP, Bolch WE, Moroz BE, Folio L. NCICT: a computational solution to estimate organ doses for pediatric and adult patients undergoing CT scans. J Radiol Prot. 2015;35:891-909. doi: 10.1088/0952-4746/35/4/891. PubMed PMID: 26609995.
20. Menzel HG, Clement C, DeLuca P. ICRP Publication 110. Realistic reference phantoms: an ICRP/ ICRU joint effort. A report of adult reference computational phantoms. Ann ICRP. 2009;39(2):1-164. doi: 10.1016/j.icrp.2009.09.001. PubMed PMID: 19897132.

21. Lee C, Lamart S, Moroz BE. Computational lymphatic node models in pediatric and adult hybrid phantoms for radiation dosimetry. Phys Med Biol. 2013;58(5):N59-82. doi: 10.1088/0031-9155/58/5/ N59. PubMed PMID: 23391692. PubMed PMCID: PMC3878984.

22. Tapiovaara M, Lakkisto M and Servomaa A. PCXMC: a PC-based Monte Carlo program for calculating patient doses in medical x-ray examinations. Report STUK-A139; Helsinki, Finland: Finnish Centre for Radiation and Nuclear Safety Authority; 1997.

23. Ahnesjö A. Collapsed cone convolution of radiant energy for photon dose calculation in heterogeneous media. Med Phys. 1989;16(4):577-92. doi: 10.1118/1.596360. PubMed PMID: 2770632.

24. McNutt T. Dose calculations: collapsed cone convolution superposition and delta pixel beam. Pinnacle White Paper No. 4535983 02474; Netherlands: Philips Medical System; 2002.

25. Wilke L, Andratschke N, Blanck O, et al. ICRU report 91 on prescribing, recording, and reporting of stereotactic treatments with small photon beams: Statement from the DEGRO/DGMP working group stereotactic radiotherapy and radiosurgery. Strahlenther Onkol. 2019;195(3):193-8. doi: 10.1007/s00066-018-1416-x. PubMed PMID: 30649567.

26. Ding GX, Munro P. Radiation exposure to patients from image guidance procedures and techniques to reduce the imaging dose. Radiother Oncol. 2013;108:91-98. doi: 10.1016/j.radonc.2013.05.034. PubMed PMID: 23830468.

27. Stock M, Palm A, Altendorfer A, Steiner E, Georg $D$. IGRT induced dose burden for a variety of imaging protocols at two different anatomical sites. Radiother Oncol. 2011;102:355-63. doi: 10.1016/j. radonc.2011.10.005. PubMed PMID: 22098793.

28. Nelson AP, Ding GX. An alternative approach to account for patient organ doses from imaging guidance procedures. Radiother Oncol. 2014;112:1128. doi: 10.1016/j.radonc.2014.05.019. PubMed PMID: 25023041.

29. Murphy MJ, Balter J, Balter S, et al. The management of imaging dose during image-guided radiotherapy: report of the AAPM Task Group 75. Med Phys. 2007;34(10):4041-63. doi: 10.1118/1.2775667. PubMed PMID: 17985650. 